\title{
La investigación sobre el espacio público en Colombia: su importancia para la gestión urbana
}

The Research on Public Space in Colombia: its Importance to the Urban Management

A pesquisa sobre o espaço público na Colômbia: sua importância para a gestão urbana

Andrea Milena Burbano*

Recibido: 15 de enero de 2014

Aprobado: 17 de marzo de 2014

Doi: dx.doi.org/10.12804/territ31.2014.08

Para citar este artículo:

Burbano, A. M. (2014). La investigación sobre el espacio público en Colombia: su importancia para la gestión urbana. Territorios, (31), 185-205. Doi: dx.doi.org/10.12804/territ31.2014.08

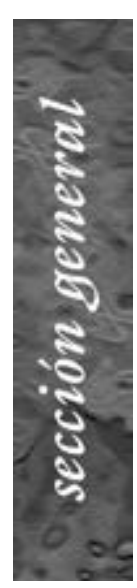

* Candidata a Doctora en Estudios Territoriales de la Universidad de Caldas (Colombia); magister en Gestión Urbana de la Universidad Piloto de Colombia y arquitecta de la Universidad de La Salle (Colombia). Profesora de la Maestría en Gestión Urbana de la Universidad Piloto de Colombia y de la Maestría en Educación de la Universidad Pedagógica Nacional. Su campo de investigación se centra en estudios territoriales, asociados con el género y la espacialidad, la movilidad, el espacio público y la calidad de vida urbana. Para la autora, el presente estudio hace parte de los avances de investigación de su tesis doctoral. Correo electrónico:arqburbano@gmail.com

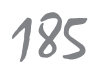


Palabras clave

Espacio público, gestión urbana, pedagogía urbana, formación ciudadana.

Keywords

Public space, urban management, urban education, citizenship education.

Palavras-chave Espaço público, gestão urbana, pedagogia urbana, formação cidadã.

\section{territarias 31}

\section{RESUMEN}

En este artículo, se presenta una primera aproximación a un estado del arte del espacio público de Colombia. Con este fin, este trabajo analiza la producción de los grupos de investigación de varias universidades e instituciones, tanto públicas como privadas. Los estudios revisados se organizaron desde las perspectivas espaciales, sociales, históricas y educativas, y se analizaron a partir de su influencia en la gestión urbana. Por último, se propone continuar con el seguimiento del desarrollo de la investigación en el campo a fin de llenar el vacío entre la investigación y las decisiones urbanas.

\section{ABSTRACT}

This paper presents a first approach to a state of the art on public space in Colombia. For this purpose, this article analyze the production of research groups from several universities and institutions, both, public and private. The reviewed studies are organized from the spatial, social, historical and educational perspectives and analyzed from their influence on the urban management. Finally, it is proposed to continue the traking of the development of research on the field in order to fill the gap between research and urban decisions.

\section{RESUMO}

Neste artigo, apresenta-se uma primeira aproximação a um estado da arte do espaço público da Colômbia. Com esse objetivo, este trabalho analisa a produção dos grupos de pesquisa de várias universidades e instituições, tanto públicas quanto privadas. Os estudos revisados se organizaram desde as perspectivas espaciais, sociais, históricas e educativas, e se analisaram a partir de sua influência na gestão urbana. Por último, propõe-se continuar com o seguimento do desenvolvimento da pesquisa no campo a fim de preencher o vazio entre a pesquisa e as decisões urbanas. 


\section{Introducción}

El espacio público en los últimos años ha sido un objeto de investigación y un elemento de discusión para distintos interesados, entre los que se encuentran los académicos, gobernantes y usuarios del espacio público en general. Ha estado presente en campañas políticas, en distintas propuestas de programas de formación ciudadana y transformación de ciudades, así como en la literatura científica y técnica que aborda el tema, aspectos estos que, en parte, se configuran como los motivos que han contribuido para que adquiriera relevancia en los procesos administrativos de las ciudades.

Dada la importancia que tiene comprender lo que ocurre en el espacio público antes de incorporarlo en los procesos que demanda la gestión urbana, en razón al impacto que suscitan las intervenciones físicas del espacio público en la sociedad y en términos generales, en el contexto urbano, es importante rastrear qué se ha investigado en torno al espacio público, como una de las formas que permite conocer su complejidad, a partir de la relación investigacióngestión, desde la que se analizan distintos elementos que enriquecen el debate del espacio público como objeto de estudio con especial énfasis en las implicaciones sobre la gestión urbana - análisis que ubica la discusión en la dimensión no visible e intangible del espacio público- y el impacto que ha generado en diversos procesos sociales de la vida actual en las ciudades.

En este sentido, los gestores del espacio público y académicos de distintas disciplinas exploran ahora el papel que este ha tenido en las ciudades, por cuanto se configura como el escenario donde se condensa la vida urbana. Al asumirse desde este sentido, entreteje facetas como la social, la económica, la gestión urbana, la estética, la individualidad, la colectividad, la historia, el ambiente natural, la comunicacional y el género. Autores como Páramo (2006) se han acercado a definir el espacio público desde esta perspectiva, que es la que se toma en cuenta en la presente investigación, la cual, además, entra en diálogo con otras, como la planteada por Licona (2007), desde la que se entiende el espacio público como el ordenador de las prácticas colectivas e individuales que expresa procesos de apropiación y se entrelaza con el modo de vida de las personas y sus diferentes formas de interacción social, mediante las cuales se exhiben las prácticas sociales que logran identificar y cohesionar los grupos culturales.

Como escenario para el encuentro o para el tránsito de las personas, se espera, el espacio público debe responder a las necesidades de quienes lo ocupan y ofrecer condiciones que busquen como finalidad el bienestar de las personas, así como aportar al conjunto de los aspectos que propician la calidad de vida del ser humano en los distintos ámbitos que hacen parte de su experiencia en la ciudad. Por todas estas razones, resulta importante explorar el desarrollo de la investigación reciente que da cuenta de la manera en que el espacio público, como objeto de estudio, ha comenzado a hacer parte de los intereses de los académicos y territarias 31

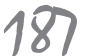


${ }^{1}$ Grupo de investigación en Gestión Urbana de la Universidad Piloto de Colombia.

\section{tersitarios 31} 188 gestores de la ciudad. Para llevar a cabo este propósito, se presenta un acercamiento a la investigación sobre espacio público llevada a cabo por investigadores en el marco de: 1) la gestión urbana, por considerar que desde esta mirada es posible agrupar investigaciones que se aproximan al estudio del espacio público a partir de distintos acercamientos disciplinares interesados de manera común en contribuir a una profundización acerca de lo que ocurre en el espacio público mediante la posibilidad de situar de forma espacial las problemáticas urbanas; 2) la pedagogía urbana, por reconocer que desde este campo de conocimiento se considera que el espacio púbico es el escenario por excelencia para la formación del ciudadano y en este se articulan distintos acercamientos e intereses disciplinares que tienen en común situar espacialmente el aprendizaje de las personas valiéndose del espacio público; y, por último, 3 ) la mirada de algunas instituciones públicas y privadas, para incorporar los aportes que en los últimos años se han realizado desde la institucionalidad, tanto pública como privada, distinta a la académica, y situada particularmente en la ciudad de Bogotá.

\section{Aproximación a la investigación sobre espacio público en el marco de la gestión urbana}

La proximidad que se toma para abordar la investigación sobre espacio público en el marco de la gestión urbana se da a partir del interés que han tenido investigadores, algunos de ellos integrantes de grupos de investigación de ciudades como Bogotá, Pereira y Cali, que se han inclinado hacia la exploración del espacio público como objeto de estudio y profundizado en la relación que existe entre los seres humanos y el hábitat urbano. Bajo este particular, se exploran las dinámicas que se suscitan en el espacio público desde la perspectiva de la gestión, el gobierno y la dimensión espacial de la ciudad ${ }^{1}$ (Documento para la renovación del registro calificado - MGU, 2007). Entre las preguntas que han orientado la exploración, están: ‘̨cuáles son esos aspectos o características que hacen eficiente al espacio público en términos de las necesidades humanas y del mejoramiento de la calidad de vida del habitante de la ciudad? ¿Qué hace eficiente al espacio público en términos de las necesidades humanas y del mejoramiento de la calidad de vida del habitante de la ciudad? ¿Qué mecanismos de participación ciudadana contribuyen a una gestión eficiente del espacio público en las ciudades? ¿Cuál ha sido el impacto económico y social del espacio público? ¿Cuáles han sido los procesos históricos de la construcción del espacio público en las ciudades? ¿Cuál ha sido su impacto en la habitabilidad urbana y en la calidad de vida de los ciudadanos? ¿Qué relación existe entre la aparición de los sociolugares y no lugares con la desaparición de la vida en público? En el marco de estas preguntas de investigación, el espacio público ha sido estudiado desde diversas miradas, entre las que se logran identificar: la socioespacial y normativa (Burbano, 2007, 2009; Sierra, 2011; Ledezma, 2005; Plazas, 2006; Pla- 
zas, 2006; Pinto, 2010, Páramo, 2011), la jurídico-normativa (Rosero, 2007; Vidal, 2008 ) y la educativa (Ocampo, 2005).

Desde la mirada socioespacial y normativa, las investigaciones enfatizan en varios aspectos, entre los que se destacan: el análisis de las experiencias, procesos y resultados de la aplicación de la herramienta de gestión social, conocida como zonas de transición de aprovechamientos autorizados (Sierra, 2011); la evaluación del impacto que se genera en los actores del espacio público al introducir los espacios análogos (Burbano, 2007); el análisis de la multiplicidad de usos del espacio público y la diversidad social que este alberga (Ledezma, 2005); la exploración de los efectos de las intervenciones sobre el espacio público sin previo análisis de las necesidades de los usuarios (Plazas, 2006); el seguimiento de los procesos en la intervención arquitectónica de los parques vecinales y de bolsillo del Distrito Capital (Plazas, 2006); la exploración sobre las posibilidades de articulación de los instrumentos de gestión urbanos frente a las necesidades y expectativas de los ciudadanos en la proyección y generación de los nuevos espacios públicos de permanencia (Pinto, 2010); el rastreo del desarrollo urbano de Bogotá para contextualizar y comprender las distintas manifestaciones sociales características de la vida en público del habitante de la ciudad de Bogotá durante el siglo Xx, mediante la presentación de aspectos urbanos y sociales destacados en la ciudad (Burbano, 2009); y el análisis de la experiencia de distintos protagonistas de la vida en público analizada bajo la perspectiva de cómo estos lugares, llamados sociolugares, vienen transformando las relaciones sociales y la vida en público en general (Páramo, 2011).

Desde la mirada o aproximación jurídico-normativa, se observa que se acentúa el interés en aspectos que tienen que ver con el seguimiento y la evaluación de los instrumentos de gestión dispuestos por la jurisprudencia urbanística colombiana, específicamente referida a los planes parciales (Rosero, 2007). En este mismo enfoque, se encuentra la investigación dirigida a explorar la norma de uso general para el diseño urbano de los diferentes equipamientos que se construyen en Bogotá (Vidal, 2008). Así también, está el estudio en el que se explora el tipo de instrumento normativo que se considera necesario implementar por parte de los entes territoriales para conciliar los derechos de propiedad privada, la función social y ecología de la propiedad en relación con el manejo de la ocupación indebida de antejardines (Rojas, 2010).

En lo que respecta a la perspectiva educativa, se ubica la exploración en acatamiento voluntario de las normas en el espacio público para contribuir a la cultura ciudadana (Ocampo, 2005). Esta investigación en particular muestra, de manera implícita en su análisis, el cuestionamiento sobre la normativa que lo regula al pretender identificar y profundizar en lo que determina que la gente que vive en Bogotá acate o no, voluntariamente, las normas de convivencia.

Si bien es cierto que en estos estudios es posible identificar la perspectiva desde territarias 31

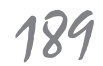


la cual ha sido abordado el espacio público, existe una constante preocupación en aportar a la calidad de vida de las personas usuarias del espacio. Estos estudios han perseguido varios propósitos y tienen como sustento la normatividad de la ciudad que rige el espacio público.

Se reconoce además en estos análisis la forma en que se asume el espacio público como escenario que cumple distintos propósitos, por ejemplo, el de situar las tensiones que se generan entre los diferentes actores por la ocupación de las ventas informales, donde el espacio público es el escenario en el que ocurren estas tensiones. Así también como el escenario que posibilita entrar en contacto con las demás personas y el entorno en sentido amplio, que, adicionalmente, permite el uso y disfrute colectivo, al igual que hacer realidad los derechos consagrados en la Constitución Política de Colombia.

Ahora bien, al revisar la manera en que se realiza el acercamiento teórico conceptual al espacio público, se destacan, en los estudios, aproximaciones a partir de categorías teóricas, centrales; una de ellas, la del concepto de lugar. Concepto que, por considerarse transversal a las disciplinas, permite el acercamiento al estudio del espacio público desde una mirada que logra abarcar las diferentes relaciones que se dan entre las personas y el ambiente. Se acentúa otra categoría teórica para el estudio del espacio público, definida bajo el concepto de reglas. En ella se mencionan los argumentos a partir de los cuales las personas acatan las normas de forma voluntaria en el espacio público en función de la cultura ciudadana. Se acotan otros conceptos para abordar la problemática en la que se inscriben los estudios sobre espacio público, entre los cuales se resaltan: capacidad de carga, apropiación del espacio público y percepción ambiental, en la medida en que el impacto de los equipamientos deportivos genere un efecto de estos tres conceptos en el espacio público. Así, también, resulta interesante encontrar la preocupación por el concepto de territorio, sus componentes, dimensiones, demandas y necesidades, para analizar las repercusiones que tiene en el reordenamiento territorial.

Sobre las maneras de aproximarse al espacio público desde el punto de vista metodológico, se puede observar que las investigaciones se valen de distintas aproximaciones de acuerdo con el criterio del investigador para acercarse de la mejor forma a la problemática de interés. Por ejemplo, el estudio de caso, en el que se combinan fuentes de información cualitativa y cuantitativa, la investigación documental, las exploraciones de campo mediante recorridos por sectores de la ciudad, el trabajo de carácter histórico documental, la investigación con enfoque etnográfico y el uso de cuestionarios, uno de ellos, el que se sustenta en la teoría de facetas, con el que se estructuran y delimitan las observaciones que guían el diseño de la pregunta de investigación.

Desde la relación entre investigación y gestión urbana, los estudios desarrollados han dado lugar a propuestas y recomendaciones que se relacionan con la gestión. 
Este es el caso de la investigación de Burbano (2007), que propone elementos de gestión que contribuyan a la proyección de espacios análogos en la ciudad de Bogotá, D.C., y formula recomendaciones de tipo físico espacial y normativo para buscar la disminución de las tensiones en los espacios públicos con características comerciales. Se encuentra también la investigación de Plazas (2006) en esta perspectiva, pues, a partir de su trabajo, da recomendaciones tendientes a la aplicación de normas referidas a los diseños de los espacios públicos vecinales, donde se reconozca la tradición social de las personas que ocupan los parques y se vinculen procesos de participación ciudadana.

De igual manera, se evidencia la relación entre el espacio público y la gestión urbana en el estudio de Vidal (2008), al derivar recomendaciones para el diseño de una normatividad general, direccionada a la organización de eventos en la ciudad y, de este modo, contrarrestar los problemas de circulación, accesibilidad, contaminación e inseguridad que se deriven de los equipamientos deportivos y de la ciudad. En algunas investigaciones, la articulación con la gestión urbana se hace explícita en la formulación del problema y los objetivos de la investigación. Esto es posible observarlo en el estudio de Rosero, que tiene como propósito "analizar las cesiones de espacio público en los planes parciales adoptados en Bogotá, con base en la política establecida por el Plan de Ordenamiento Territorial y el Plan Maestro de Espacio Público" (Rosero, 2007, p. 13).
El estudio de Pinto se ubica en esta perspectiva al centrarse en "valorar el nivel de correspondencia entre las necesidades, las expectativas de los ciudadanos y el diseño espacial de los espacios públicos (...)" (Pinto, 2010, p. 18) y, a partir de ello, aportar insumos para formular elementos de gestión que tengan en cuenta estos niveles de correspondencia. De la misma forma, se puede ubicar el estudio que busca establecer, dentro del marco de la gestión urbana, el diseño conceptual de un instrumento que le permita a los entes territoriales dar solución al problema presentado por la ocupación indebida de las áreas de antejardín y proponer la conceptualización de un instrumento de gestión urbanística (Rojas, 2010).

Otra manera de asumir la articulación con la gestión urbana, distinta a la de la intervención tangible en el territorio, se encuentra en investigaciones que se preocupan por esa fase que se puede considerar anterior a la intervención física del espacio público. En este grupo de investigaciones, se ubica la de Ocampo (2005), que se preocupa por ver la gestión urbana desde el acatamiento voluntario de normas en el espacio público en Bogotá. El estudio de Ledezma (2005) se suma a esta perspectiva, al considerar que la gestión urbana, además de profundizar en los problemas urbanos relacionados con la vivienda, el transporte, entre otros temas urbanos, es necesario que profundice en las particularidades del espacio público de las ciudades que, según el autor, se logran recoger mediante una aproximación sociológica al estudio de territarias 31

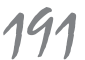


${ }^{2}$ En el contexto colombiano, se destacan las investigaciones sobre espacio público desarrolladas en la Fundación Universitaria Autónoma de Colombia, en la ciudad de Bogotá.

${ }^{3}$ En el contexto colombiano, resaltan las investigaciones sobre espacio público llevadas a cabo en la Universidad Católica Popular de Risaralda.

${ }^{4}$ En el contexto colombiano, sobresalen las investigaciones sobre espacio público generadas en la Universidad de San Buenaventura de la ciudad de Cali.

${ }^{5}$ En el contexto colombiano, se destacan las investigaciones sobre espacio público elaboradas por parte delgrupo de investigación denominado Pedagogía Urbana y Ambiental de la Universidad Pedagógica Nacional, de la ciudad de Bogotá.

\section{territarias 31} 192 las prácticas sociales y culturales situadas en el espacio público, aproximarse a los distintos usos que tiene para contribuir en la planificación de estos y, de esta forma, incidir en las políticas urbanas relacionadas con el espacio público. Por su parte, Plazas (2006), al sintetizar en cuestionamientos la manera en que la gestión urbana tendría que abordar el diseño y transformaciones espaciales de los espacios públicos, propone interrogarse por lo que se entiende respecto a las necesidades urbanas colectivas, el tipo de personas que están en capacidad de decidir sobre ese tipo de necesidades y el tipo de norma que se toma como base para determinar que las necesidades de un grupo poblacional se mantengan vigentes ante la transformación espacial de los parques, tomando como referencia la plazoleta de Lourdes de la ciudad de Bogotá.

Se suma a estas investigaciones, la de Gómez (2003), quien reúne una serie de ensayos en los que se realiza una lectura de la vida cotidiana de distintos lugares que conforman el centro de la ciudad de Bogotá y los usos y prácticas sociales que allí se desarrollan. ${ }^{2}$ Así también, las de Yory (2002), al hablar sobre el monumento y señalar la necesidad de formular un nuevo proyecto político que contemple un nuevo pacto entre el bien patrimonial y la ciudad.

Por su parte, Parra y colaboradores $(2010)^{3}$ exploran el espacio público en relación con el comercio en la calle y resaltan, entre otros aspectos, el papel de la ciudadanía como el actor más importante frente al Estado y los comerciantes que se encuentran en el espacio público de Pereira.
Los autores presentan algunas conclusiones derivadas del análisis del registro de vendedores informales en las zonas del centro y subcentro de la ciudad, donde se comenta sobre las aptitudes laborales, de formación, y expectativas de empleo de los vendedores informales y se finaliza con la sistematización del Plan de Manejo Integral de Ventas Informales en la ciudad de Pereira.

En esta misma dirección, se sitúan los trabajos en los cuales se explora el comportamiento ciudadano, la educación política, las formas urbanas arquitectónicas (Salcedo, 2010a) del espacio público en la ciudad de Cali, así como la propuesta de asumirse como una temática en las ciencias sociales y humanas (Salcedo, 2008a, 2008b), y el estudio de la cotidianidad dramática en el espacio público (Salcedo, 2010b), que aportan a la reflexión teórica sobre el campo referido al espacio público. ${ }^{4}$

\section{Aproximación a la investigación de espacio público en el marco de la pedagogía urbana}

Como se ha mencionado anteriormente, otro de los marcos que se toma para hacer una primera aproximación a la investigación sobre espacio público en Colombia es el de la pedagogía urbana, ${ }^{5}$ entendida co- $^{-}$ mo el cuerpo de conocimiento que integra la teoría, los conceptos y las experiencias urbanas, orientados a la formación del ciudadano y al fortalecimiento de la identidad urbana mediante mecanismos no formales en el ámbito de la ciudad (Páramo, 2010). Este acercamiento se lleva a cabo desde los 
estudios que han adelantado investigadores que asumen el espacio público como escenario educativo, a partir del cual se otorga importancia al significado que tiene la vida pública y de encuentro con los otros, que moldea los comportamientos de las personas en la medida en que funciona como un conjunto de oportunidades para el aprendizaje (Páramo, 2004).

Cabe mencionar que diversas instituciones educativas y de investigación, así como político-administrativas, dan cuenta de experiencias relacionadas con la pedagogía urbana. Algunas de ellas han generado una marcada influencia, tanto en los sistemas educativos como en la forma de concebir la ciudad y la educación. Un ejemplo por mencionar es el movimiento de Ciudad Educadora, el cual busca facilitar el intercambio y difusión de las acciones educativas innovadoras en el medio urbano, que cuenta con el soporte de la Carta de ciudades educadoras propuesta en el I Congreso Internacional de Ciudades Educadoras, efectuado en Barcelona en 1990. Ciudad Educadora, por lo general, se concibe a partir del compromiso de las instituciones y del marco político-administrativo de una ciudad, que implica una proyección y una voluntad de la administración de la ciudad para que se oriente y se dé continuidad a una política pública soportada por planes, programas y proyectos de educación, formal, no formal e informal, para hacer educadora la ciudad (Páramo, 2010b).

A una escala más local, se puede decir que el interés de pensar en la educación de la ciudadanía, así como en el fortalecimien- to de la ciudad como medio o como objeto de educación, se ha convertido igualmente en eje político de recientes administraciones en el Distrito Capital de Bogotá. Es de recordar, por un lado, la primera administración de Antanas Mockus (1995-1998), cuyo plan de gobierno se llamaba 'Formar ciudad', desde el cual se desarrolló el programa de Cultura Ciudadana; y, por otro, la administración de Luis Eduardo Garzón (2005-2007), la cual adelantó el programa educativo denominado 'Bogotá, una gran escuela' (Documento de trabajo grupo Pedagogía Urbana y Ambiental, en prensa).

Desde la perspectiva de la pedagogía urbana, los interrogantes que han orientado la exploración del espacio público son: ¿cuál es el papel que juega el espacio público en la formación del ciudadano? ¿Cuáles han sido los lugares públicos significativos para la mujer en Bogotá a lo largo de su historia? ¿Qué papel ha jugado el espacio público en el sostenimiento de las prácticas sociales que han caracterizado la vida en público a lo largo de la historia? ¿Qué prácticas sociales han estado asociadas al espacio público de la ciudad? ¿Qué transmiten las obras de arte a los ciudadanos en el espacio público? ¿Qué papel juega el espacio público en la percepción de la calidad de vida urbana percibida? ¿Cuáles son los usos que hacen los habitantes de una ciudad de sus lugares públicos? ¿Cómo se conducen y negocian las relaciones en el espacio cuando están rodeados no por personas cercanas, sino por extraños? ¿De qué manera los centros comerciales han desplazado a las plazas y calles en la facilitación de los encuentros territarias 31

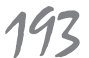


sociales? ¿Son estos encuentros similares a los que se daban en lugares públicos abiertos? ¿Qué mecanismos de participación pedagógica son necesarios para conseguir que las personas se apropien del espacio? ¿Qué papel cumple la pedagogía al hacer los espacios públicos menos segregacionistas y más incluyentes? ¿Qué aspectos pedagógicos se deben tener en cuenta en el Plan Maestro de Espacio Público para la ciudad? y ¿qué conoce la comunidad acerca de las reglas de convivencia en el espacio público? En el marco de estos cuestionamientos, el espacio público ha sido estudiado desde diversos enfoques, entre los que se logra identificar: el histórico (Páramo \& Cuervo, 2006; Páramo \& Cuervo, 2009; Parra, 2007; Arango, 2007; Castaño, 2009; Alarcón, 2009); educativo (Moreno, 2009; Melo, 2009; Rincón, 2010; Cuesta, 2010; Rico, 2011); educativo y cultural (Espinosa, 2009); social (Parra \& Torres, 2007; Castellanos, 2010; Villamizar \& Rondón, 2007; Montoya, 2011); socioespacial (Gómez, 2010); y normativo (Montoya, 2010).

Los estudios que se ubican en el enfoque histórico exploran distintos aspectos, entre los que se destacan, la historia de las prácticas sociales situadas en el espacio público de la ciudad de Bogotá desde su fundación hasta el siglo XIX, así como la experiencia urbana de los bogotanos durante el siglo Xx en el espacio público (Páramo \& Cuervo, 2006, 2009). Sumadas a estas investigaciones, se encuentran otras que dan lugar a exploraciones acerca de los usos del espacio público en la ciudad, a partir de una mirada histórica, desde las prácticas sociales y la memoria colectiva, en el período de 1910 a 1948 (Parra, 2007). En esta misma dirección, se encuentra el estudio sobre el significado de la imagen histórica del parque General Santander para los habitantes de la ciudad de Florencia, con el que se busca determinar los significados y las conceptualizaciones que transmiten a los habitantes de la ciudad (Arango, 2007).

Así también, se ubican exploraciones acerca de la experiencia en el espacio público registrada en las imágenes fotográficas de Bogotá en el siglo Xx, la cual tiene como objetivo caracterizar las funciones que cumplen los lugares, eventos, acontecimientos y protagonistas asociadas a los usos cotidianos de los lugares públicos a través de la identificación de prácticas sociales situadas (Castaño, 2009); y el estudio de la mujer en el espacio público en Bogotá, en el período de 1910 a 1948, mediante el que se propone identificar los lugares públicos y la manera de ser significados por parte de la mujer en la ciudad de Bogotá (Alarcón, 2009).

Al analizar el enfoque educativo en las investigaciones, se observa que este se encuentra orientado de distintas maneras. Así, por ejemplo, están los estudios que exploran el acatamiento de reglas de convivencia en el espacio público. En esta dirección, se ubica el estudio de las reglas que las mujeres y los hombres identifican y siguen en algunos lugares públicos de la ciudad de Bogotá, con el fin de establecer algunos parámetros que se puedan aplicar para el fortalecimiento de las competencias ciudadanas (Moreno, 2009a). Igualmen- 
te, la puesta a prueba de un conjunto de reglas representadas iconográficamente en señales educativas que generen la ocasión de motivar en los ciudadanos prácticas de convivencia en el espacio público. Otras investigaciones que se ubican en este componente educativo exploran en torno a la experiencia urbana. En estas se encuentra la investigación que se interesa por establecer la manera en que inciden los procesos de identificación, apropiación y apego en la experiencia urbana de lugar, así como en la formación ciudadana (Melo, 2009), y por caracterizar los aprendizajes que adquieren los jóvenes de un colegio distrital de la ciudad de Bogotá a partir de su experiencia directa en espacios públicos de la localidad en la que habitan (Rincón, 2010).

Por último, en este componente se ubica el estudio que busca analizar la función que cumple la educación patrimonial en la formación del ciudadano, tomando el caso de la preservación del Parque de la Independencia de la ciudad de Bogotá como bien patrimonial (Rico, 2011). Se suma a este enfoque, con un componente cultural, el estudio de Espinosa (2009), el cual explora el arte en el espacio público de Bogotá como elemento educador. Investigación que se centra en el estudio del apego al lugar desde la perspectiva urbana de Bogotá y en identificar de qué forma las obras de arte plástico, en el espacio público de Bogotá, representan oportunidades de aprendizaje a partir de la conceptualización que hacen los habitantes de ellas.

Se sitúa en el enfoque social la investigación que explora el proceso de construc- ción social del espacio público (Parra \& Torres, 2007). De igual manera, la investigación sobre identidad social y de lugar en jóvenes de la localidad cuarta, ubicada en el suroriente de Bogotá, cuyo propósito es describir las representaciones sociales que definen la filiación a grupos sociales en relación con el lugar urbano y su valor educativo en la construcción y fortalecimiento de la identidad social urbana en jóvenes de la mencionada localidad (Castellanos, 2010). Hacen parte del mismo enfoque social el estudio acerca del proceso de construcción social y de las significaciones de las personas sobre el espacio público de la Plaza de Mercado La Concordia de la ciudad de Florencia (Caquetá) (Villamizar \& Rondón, 2007), el estudio sobre el apego al lugar desde la perspectiva urbana de Bogotá (Montoya, 2011) y el que indaga acerca de la identidad de lugar del bogotano a partir de la interacción con el pasado de la ciudad en el espacio público (Páramo, 2002). En este componente, se ubica el trabajo compilado por Páramo y García (2010) en torno a la dimensión social del espacio público a partir de la mirada a las distintas formas de apropiación, relación y uso de los espacios públicos urbanos y sus implicaciones para la calidad de vida y la convivencia entre extraños, desde distintas perspectivas: la comunicación, el género, la pedagogía y la psicología ambiental.

En los estudios que acentúan en mayor medida el enfoque socioespacial, se ubica la exploración sobre la literatura científica de género y espacialidad, la cual se encuentra enmarcada en tres perspectivas diferentes: territarias 31

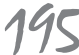


las representaciones sociales y usos diferenciados del espacio, la división de roles en los espacios públicos y privados, y la planeación urbana del espacio (Páramo \& Burbano, 2011). Así también, la que explora la experiencia de la mujer en el espacio público a partir de su rol social (Páramo \& Burbano, 2007) y sobre la experiencia urbana de los estudiantes de un colegio en la ciudad de Bogotá (Gómez, 2010).

En el enfoque normativo, se logra ubicar el estudio que explora los componentes educativos de la normatividad para consolidar una pedagogía urbana en Bogotá, la cual indaga acerca de los componentes de la legislación vigente para el uso del espacio público y las estrategias pedagógicas que se pueden formular a partir de ellos, con miras a lograr la cultura ciudadana (Montoya, 2010).

En las exploraciones realizadas, se observa la preocupación por asumir una definición de espacio público que contemple otros aspectos además de los que se examinan desde la norma. Por lo que se sugiere desde esas investigaciones que, al asumir el espacio público como categoría, se incorporen conceptos de identidad de lugar, historia, simbolismo, además de elementos psicológicos y sociales de los habitantes, que contribuyen a determinar sus relaciones y formas de vivir (Espinosa, 2009). Se llama la atención sobre la necesidad de considerar el espacio público como escenario para la comunicación (Páramo, 2002) y, de esta manera, contribuir a la formación ciudadana en un escenario donde las personas aprendan reglas para relacionarse con los otros y con el mismo ambiente físico (Páramo \& Cuervo, 2006; Burbano \& Páramo, 2008; Burbano, 2010a, 2010b); escenario para la implementación de contingencias sociales orientadas a la creación y sostenimiento en el tiempo de prácticas culturales que contribuyan a la convivencia entre los ciudadanos (Páramo, 2010a). El trabajo de Cuesta (2010) pone a prueba estos planteamientos en un experimento de campo en el espacio público de Bogotá.

Autores como Rincón (2010) acercan la definición de espacio público al concepto de lugar, en el que se identifica una dimensión sociocultural a partir de la cual se le considera como lugar de relación, identificación, encuentro entre las personas, movimiento y sentido comunitario. Se observa que se equiparan, en el mismo nivel teórico, los conceptos de espacio y lugar para construir la definición de espacio público. En este sentido, Alarcón (2009) lo asume como el lugar donde se precisa la responsabilidad ciudadana. Por su parte, Arango (2007) se suma a la intención por reconocer el valor cultural que tiene el concepto como integrador del hombre como ser vivo y social. Como se puede vislumbrar, estos trabajos dan cuenta de experiencias relacionadas con una pedagogía urbana e intentan interrelacionar los campos de la educación y la ciudad a partir del concepto de espacio público como escenario para aprender.

Se suman a la intencionalidad de aportar a la discusión del espacio público desde el marco de la pedagogía urbana, algunos estudios que reflexionan sobre la ciudad en general, con acento en temáticas urbanas 
que se consideran importantes y que son abordadas pedagógicamente, relacionadas con los procesos educativos y de aprendizaje, aspectos históricos, estéticos, de comunicación, redes urbanas y desarrollos tecnológicos y culturales, entre otros ${ }^{6}$ (Bustamante \& Aranguren, 2007). En esta misma dirección, se ubican los desarrollos de investigación orientados a contribuir en la construcción de un marco teórico desde el cual se haga posible la formulación de proyectos concertados de ciudad que involucren la formación ciudadana en las actividades que demandan la intervención, uso y administración del espacio público. Estudios como este se orientan a pensar la ciudad desde la formación ciudadana y de los desafíos y propuestas para la construcción de un proyecto concertado de ciudadanía, mediante la intervención del espacio público, considerado este como escenario análogo a la participación social y política, comprometido con el mejoramiento de una calidad de vida (Yory, 2007). ${ }^{7}$

\section{Un acercamiento a la investigación sobre espacio público a partir de la mirada de algunas instituciones públicas y privadas de la ciudad de Bogotá}

Por último, se considera importante, como aporte a la aproximación al estado del arte del espacio público de Colombia, incorporar las contribuciones que desde los últimos años se han realizado desde la institucionalidad, tanto pública como privada, distinta a la académica. En esta dirección, desde el año 2001, Bogotá se ha valido de una encuesta que le ha permitido indagar acerca de la cultura ciudadana de la ciudad. Proceso que ha sido apoyado por el Observatorio de Culturas, en sus inicios llamado Observatorio de Cultura Urbana, creado en 1978 y adscrito al Instituto de Cultura y Turismo del Distrito.

El principal objetivo de esta institución ha estado orientado, desde un principio, a consolidarse como el principal motor de construcción, planeación y ejecución de las políticas culturales (Pecha, 2006). Ha llevado a cabo el análisis del comportamiento ciudadano mediante mediciones y sondeos (Secretaría de Cultura, Recreación y Deporte, Observatorio de Culturas), con el fin de ponerlo a disposición de la academia, el gobierno y la propia ciudadanía para la constitución de políticas generales y específicas, principalmente en los temas relacionados con cultura, recreación y deporte (Secretaría de Cultura, Recreación y Deporte, ¿Qué es el Observatorio de Culturas?).

En términos generales, las distintas versiones de las encuestas, con diferentes nominaciones y énfasis en su contenido, de acuerdo con la administración distrital interesada en recoger la información, han sido llevadas a cabo en cada período de gobierno para observar los cambios culturales en ciclos entre períodos de gobierno. Los resultados obtenidos se han considerado como insumo importante en el diseño, formulación y seguimiento de actividades y políticas públicas.

La primera encuesta, cuyo propósito estuvo orientado a fortalecer la cultura
${ }^{6}$ Estudios que se encuentran organizados en torno a la mirada de las "Pedagogías urbanas desde la escuela", trabajo adelantado en convenio interinstitucional entre la Secretaría de Educación Distrital y la Universidad Distrital Francisco José de Caldas.

${ }^{7}$ Para aportar en la discusión sobre el espacio público desde la investigación, se tiene en cuenta el trabajo adelantado por el grupo denominado Pensar la Ciudad, de la Pontificia Universidad Javeriana, de la ciudad de Bogotá.

tersitarios 31

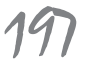


ciudadana, se lleva a cabo en el año 2001. Posteriormente, con base en la primera encuesta, se recoge información en el año 2003 y se profundiza en los procesos de investigación desde la mirada de la convivencia, la cultura política, el arte y el patrimonio. En el año 2005, a partir de los mismos ejes de la encuesta anterior, se indaga acerca de las normas de tránsito, normas de espacio público, y se hace énfasis en la convivencia ciudadana. En el año 2007, la encuesta se enfoca en la apropiación de los derechos por parte de hombres y mujeres en Bogotá. En 2008, indaga en torno a los sistemas reguladores del comportamiento, la movilidad, la tolerancia, la cultura tributaria, entre otros temas.

En el año 2011, la sexta versión de la encuesta gira alrededor de la consolidación de un nuevo concepto de indagación, basado en la cultura democrática, y examina la percepción de las personas sobre la calidad de vida. Esta última explora las actividades físicas, el deporte y la recreación, específicamente con respecto a lo agradables, limpios, seguros, amplios y bien equipados que resultan los parques y los espacios públicos de uso recreativo cercanos a la residencia del encuestado (Secretaría de Cultura, Recreación y Deporte, 2011).

La Secretaría de Cultura, Recreación y Deporte, por medio de su Observatorio de Culturas, efectúa mediciones de eventos que llevan a cabo sus direcciones y las entidades adscritas al sector, entre los que se destacan los festivales al parque: Hip Hop al Parque, Ópera al Parque, 'Rock' al Parque, Colombia al Parque, Salsa al Parque, $\mathrm{Mu}$ - jeres al Parque y 'Jazz' al Parque; y otros eventos, como la Marcha LGBTI y el SeptimAfro (Secretaría de Cultura, Recreación y Deporte, 2010).

La preocupación sobre la ciudad y la ciudadanía como fenómenos culturales hace parte de los intereses por explorar en las acciones promovidas por la administración distrital en el marco del programa de Cultura Ciudadana. En este contexto, se ubica la investigación de López (2003) sobre políticas culturales orientadas al plano de la vida cotidiana a partir de la evaluación de las estrategias de comunicación del programa de Cultura Ciudadana entre los años 1995 y 1997. En la mencionada investigación, se definen los trayectos comunicativos de Cultura Ciudadana en Bogotá, de los que hace parte el uso adecuado y el disfrute del espacio público urbano. Se suman a este tipo de exploraciones los trabajos realizados por Zambrano y el Museo de Desarrollo Urbano de Bogotá (2001), Saldarriaga y sus colegas (1998), y Mejía y Zambrano (2000), estos últimos en su edición, con la participación del Observatorio de Cultura Urbana, dan evidencia del interés que tiene la Alcaldía Mayor de Bogotá por acercarse al estudio de la ciudad.

Con el propósito de aportar al desarrollo y la gestión urbana de la ciudad, se han adelantado estudios por parte de entidades privadas sin ánimo de lucro, como la Cámara de Comercio de Bogotá, que busca promover un entorno adecuado para el desarrollo económico y productivo de la ciudad y la región. Sobre este particular ha abordado temas de reactivación económica 
ligados al desarrollo económico local de áreas estratégicas de la ciudad, como la carrera 15, el Eje Ambiental de la Avenida Jiménez y La Candelaria; ha revisado experiencias internacionales de recuperación de centros urbanos y marketing de ciudad; ha caracterizado y concebido propuestas con distinto alcance, tanto regional como mundial, en zonas como el Anillo de Innovación y el entorno del Aeropuerto Internacional Eldorado (Cámara de Comercio de Bogotá, Desarrollo y gestión urbana).

Con el propósito de dar a conocer los avances de política pública sobre aprovechamiento económico, esta entidad ha desarrollado eventos, como el Foro de Espacio Público y Ciudad, y publicaciones, como la del Observatorio de Aprovechamiento Económico de Espacio Público de Bogotá, con énfasis en ventas callejeras y el espacio público como motor de desarrollo económico local, entre otras, dirigidas a empresarios, organizaciones sociales, autoridades, academia y ciudadanía (Cámara de Comercio de Bogotá, 2012b). De igual manera, se ha preocupado por liderar proyectos como el denominado "Centro Hábitat Urbano", a partir del cual formula propuestas para el desarrollo de áreas estratégicas de la ciudad desde los componentes social, ambiental, urbanístico e inmobiliario (Cámara de Comercio de Bogotá, 2012a).

\section{A manera de conclusión}

Como puede apreciarse, por la revisión de la literatura, el espacio público ha sido explorado desde distintas miradas, entre las que se encuentran: la espacial, social, cultural, normativa, jurídica, histórica y educativa. El interés que ha despertado el estudio del espacio público por parte de distintos grupos de investigación de universidades del país responde, en gran parte, al papel protagónico que ha logrado en las ciudades. La literatura revisada da cuenta de ello, dado que muestra las diferentes maneras en que ha sido abordado: como el escenario para el uso y disfrute colectivo, en el que se presenta una diversidad de comportamientos entre conocidos y desconocidos, lo cual podría desenlazar una serie de tensiones o conflictos entre quienes hacen presencia en el espacio público, producto de la diversidad de intereses que persiguen quienes lo ocupan y buscan cumplir distintos propósitos.

En parte, se puede decir que esto ha conducido a que se indague sobre el acatamiento de las normas de convivencia ciudadana en el espacio público y sea este uno de los aspectos sobre el cual se problematice. Ha sido visto como el escenario propicio para que las personas se formen mediante la adquisición de distintos tipos de aprendizajes que facilita el medio urbano, lo que ha conducido a que se problematice alrededor de los mecanismos de educación ciudadana y la función que cumple la educación patrimonial en la formación del ciudadano. Ha sido explorado por considerarse como el escenario para la interacción, la producción de narrativas, el encuentro, así como elemento articulador en la valoración de la calidad de vida en las ciudades contemporáneas. territarias 31

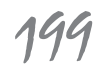


Así también, por asumirse como el espacio análogo a la participación social y política, y escenario donde se disputan los derechos consagrados en la Constitución Política de Colombia. Estas formas en que ha sido reconocido el espacio público en la investigación han conducido a que se problematice en torno a distintas dinámicas que en él se generan, algunas de ellas producto de las variadas maneras de ocupación que se dan en el espacio público, así como de las formas de participación que las personas pueden tener en los procesos que demanda la intervención física de estos espacios.

Se ha problematizado en torno a las condiciones, no solo físicas, que se espera reúnan los espacios públicos para que respondan a las necesidades de las personas, así como por su ubicación en la estructura urbana de las ciudades. Sumado a esto, ha existido una preocupación por indagar sobre las posibilidades de articulación de los instrumentos de gestión urbanos presentes en el Distrito Capital, con las necesidades y expectativas de los ciudadanos en los espacios públicos. El estudio sobre el impacto que ha generado la normatividad respecto al uso del espacio público en la actualidad, a partir de una mirada histórica de las prácticas sociales, ha sido de interés para problematizar. Asociado a los usos cotidianos de los espacios públicos, se encuentra el estudio de las funciones que cumplían los lugares, eventos, acontecimientos y protagonistas en la historia de la ciudad. Se suman a estas exploraciones los procesos de identificación, apropiación y apego que inciden en la experiencia urbana del espacio público, al igual que los procesos de construcción social e identidad social.

Continuar con la exploración del desarrollo de la investigación reciente para dar cuenta de la manera en que el espacio público, como objeto de estudio, ha hecho parte de los intereses de los académicos y gestores de la ciudad, así como investigar en torno a este, en el marco de las dinámicas urbanas actuales frente a procesos de sostenibilidad, desarrollo, conflicto, descentralización y globalización, se considera importante por tener en cuenta como parte de los intereses de investigación, por estimarse que son aspectos que inciden en las decisiones sobre gestión urbana y necesitan explorarse en el medio colombiano.

A partir de ello, contribuir, por una parte, a la discusión teórica contemporánea sobre el espacio público en el ámbito latinoamericano y, por otra, al análisis de las problemáticas que ocurren en las ciudades en razón a las repetidas intervenciones físicas de sus espacios públicos, lejanas de un conocimiento y comprensión de las distintas dinámicas que se suscitan en ellos. Por este motivo, se propone no abandonar la secuencia ‘investigación-gestión' del espacio público para hacer énfasis en la necesidad de conocer y comprender lo que ocurre en el espacio público antes de incorporarlo en los procesos que demanda la gestión urbana, debido al impacto que suscitan las intervenciones físicas del espacio público en la sociedad y, por lo tanto, en el desarrollo urbano de la ciudad. 
Para lograr este objetivo, se hace necesario continuar fortaleciendo los procesos de investigación, no solo desde las universidades, sino desde las instituciones, tanto públicas como privadas, que tienen injerencia sobre el espacio público, para lo cual los grupos de investigación requieren realizar alianzas con organismos nacionales e internacionales que permitan la financiación de proyectos, la socialización de los resultados de las investigaciones y la realización de estudios que demanden la comparación de las experiencias entre distintos países en torno al estudio del espacio público.

\section{Referencias}

Alarcón, M. B. (2007). Lugares significativos para la mujer en Bogotá, de 1910 a 1948. Revista Pre-til, (13), 8-28.

Arango, M. (2007). Lectura de los lugares significativos del espacio público a través de la imagen en el Parque Santander de la ciudad de Florencia (Tesis de grado, Universidad Pedagógica Nacional, Bogotá, Colombia).

Barker, R. G. (1968). Ecological psychology: concepts and methods for studying the environment of human behavior. Stanford, CA: Stanford University Press.

Burbano, A. M. (2007). Impacto de las modificaciones espaciales entre actores del espacio público. Revista Pre-til, (16), 80-92.

Burbano, A. M. (2009). Contexto sociourbano del espacio público de la ciudad de Bogotá en el siglo xx. En P. Páramo, La experiencia urbana en el espacio público de Bogotá en el siglo XX. Una mirada desde las prácticas sociales (pp. 21-49). Bogotá: Ediciones Universidad Pedagógica Nacional.

Burbano, A. M. (2010a). La convivencia ciudadana: su análisis a partir del 'aprendizaje por reglas'. Revista Colombiana de Educación, (57), 28-45.

Burbano, A. M. (2010b). Los manuales de convivencia: intencionalidad y mecanismos que establecen para regular el comportamiento de los ciudadanos en escenarios colectivos. En P. Páramo, La dimensión social del espacio público: aportes para la calidad de vida urbana (pp. 131-143). Ediciones Universidad Pedagógica Nacional y Universidad Santo Tomás.

Burbano, A. M. (2011). Género y sociolugares. En P. Páramo, Sociolugares (pp. 128-135). Bogotá: Ediciones Universidad Piloto de Colombia.

Burbano, A. M., \& Páramo, P. (2008). El aprendizaje por reglas y la convivencia ciudadana en el espacio público. Revista Pre-til, (18), 62-72.

Bustamante, B., \& Aranguren, F. (Comps.). (2007). Pedagogias urbanas. Bogotá: Alcaldía Mayor de Bogotá.

Cámara de Comercio de Bogotá. (2012a). Centro de Hábitat Urbano. Recuperado de http://camara.ccb.org.co/contenido/contenido.aspx? catID $=128 \&$ conI $\mathrm{D}=726$ \&pagID $=460$

Cámara de Comercio de Bogotá. (2012b). Espacio público. Recuperado de http:// camara.ccb.org.co/contenido/contenido. $\operatorname{aspx}$ ?conID $=983 \&$ cat ID $=86$ territarias 31 201 
Cámara de Comercio de Bogotá. (s. f.). Desarrollo y gestión urbana. Recuperado de http://camara.ccb.org.co/contenido/contenido.aspx? catID $=74 \&$ con $\mathrm{ID}=2888$

Canter, D. (1991). Understanding, assessing, and acting in place: is an integrative framework possible? En T. Garling \& G. Evans (Comps.), Environment, cognition and action (pp. 191-209). New York: Oxford.

Castaño, C. (2010). La experiencia en el espacio público registrada en las imágenes fotográficas de Bogotá en el siglo XX (1910-1948): una mirada histórica desde las prácticas sociales (Tesis de grado, Universidad Pedagógica Nacional, Bogotá, Colombia).

Castellanos, F. (2010). En busca de identidad social y de lugar en jóvenes: localidad cuarta de Bogotá (Tesis de grado, Universidad Pedagógica Nacional, Bogotá, Colombia).

Cuesta, O. (2010). Señalización educativa para la convivencia en el espacio público (Tesis de grado, Universidad Pedagógica Nacional, Bogotá, Colombia).

Espinosa, D. (2009). Arte en el espacio público como elemento educador (Tesis de Maestría en Educación, Universidad Pedagógica Nacional, Bogotá, Colombia).

Gómez, J. P. (2010). La experiencia urbana en los estudiantes del Colegio Minuto de Dios: un acercamiento a la perspectiva de multilugares en la ciudad de Bogotá (Tesis de grado, Universidad Pedagógica Nacional, Bogotá, Colombia).
Gómez, N. A. (2003). El Centro: fragmentos de la vida callejera. Bogotá: Universidad Autónoma de Colombia.

Grupo de investigación Pedagogía Urbana y Ambiental (en prensa). Documento de trabajo.

Ledezma, E. (2005). Uso y aprovechamiento del espacio público en los sectores de Chapinero y Centro de Bogotá, D.C.: un análisis sociológico (Tesis de grado, Universidad Piloto de Colombia, Bogotá, Colombia).

Licona, E. (2007). Habitar y significar la ciudad. Puebla: Conacyt y Casa Abierta al Tiempo.

López, L. (2003). Construir ciudadanía desde la cultura. Aproximaciones comunicativas al programa de Cultura Ciudadana (Bogotá, 1995-1997). Bogotá: Alcaldía Mayor de Bogotá, Instituto Distrital de Cultura Urbana, Consejo Latinoamericano de Ciencias Sociales.

Maestría en Gestión Urbana (en prensa). Documento para la renovación del registro calificado.

Mejía, P. G., \& Zambrano, F. (Eds.). (2000). La ciudad y las ciencias sociales. Ensayos y aproximaciones. Bogotá: Centro Editorial Javeriano, Instituto Distrital de Cultura y Turismo.

Melo, N. (2009). La experiencia urbana de las culturas juveniles: los skinheads. Pretexto para una pedagogía urbana (Tesis de grado, Universidad Pedagógica Nacional, Bogotá, Colombia).

Montoya, L. (2010). Componentes educativos de la normatividad para consolidar una pedagogía urbana en Bogotá, D.C. (Tesis 
de grado, Universidad Pedagógica Nacional, Bogotá, Colombia).

Montoya, N. (2011). El apego al lugar desde la experiencia urbana de Bogotá (Tesis de grado, Universidad Pedagógica Nacional, Bogotá, Colombia).

Moreno, Z. (2009a). Las competencias ciudadanas y las reglas de los lugares vividas por hombres y mujeres (Tesis de grado, Universidad Pedagógica Nacional, Bogotá, Colombia).

Moreno, Z. (2009b). Las reglas de los lugares: una cuestión de interpretación (Tesis de grado, Universidad Pedagógica Nacional, Bogotá, Colombia).

Ocampo, A. (2005). La gestión urbana en el acatamiento voluntario de normas en el espacio público (Tesis de grado, Universidad Piloto de Colombia, Bogotá, Colombia).

Páramo, P. (2002). En busca de la identidad de lugar del bogotano: interacción con el pasado de la ciudad en el espacio público. Revista Territorios, 8, 63-84.

Páramo, P. (2004). Algunos conceptos para una perspectiva optimista de vivir la ciudad. Revista Territorios, 10-11,91-109.

Páramo, P. (2010a). Aprendizaje situado: creación y modificación de prácticas sociales en el espacio público urbano. Psicologia \& Sociedade, 22, 130-138.

Páramo, P. (2010b). Pedagogía urbana: elementos para su delimitación como campo de conocimiento. Revista Colombiana de Educación, (57), 14-27.

Páramo, P. (2011). Sociolugares. Bogotá: Ediciones Universidad Piloto de Colombia.
Páramo, P., \& Burbano, A. M. (2007). La experiencia de la mujer en el espacio público a partir de su rol social. Revista Pre-til, (13), 8-27.

Páramo, P., \& Burbano, A. M. (2011). Género y espacialidad: análisis de factores que condicionan la equidad en el espacio público urbano. Universitas Psychologica, 10(1), 61-70.

Páramo, P., \& Cuervo, M. (2006). Historia social situada en el espacio público de Bogotá. Bogotá: Ediciones Universidad Pedagógica Nacional.

Páramo, P., \& Cuervo, M. (2009). La experiencia urbana en el espacio público de Bogotá en el siglo XX: una mirada desde las prácticas sociales. Bogotá: Ediciones Universidad Pedagógica Nacional.

Páramo, P., \& García, M. (2010). La dimensión social del espacio público: aportes para la calidad de vida urbana. Ediciones Universidad Pedagógica Nacional y Universidad Santo Tomás.

Páramo, P., Arias, J. D., Melo, H., \& Pabón, P. (1999). Apreciación del paisaje. Nuestros vínculos con la naturaleza. Bogotá: Universidad Pedagógica Nacional.

Parra, L. (2007). Los usos del espacio público en Bogotá de 1910 a 1948: una mirada histórica desde las prácticas sociales y la memoria colectiva (Tesis de grado, Universidad Pedagógica Nacional, Bogotá, Colombia).

Parra, M., \& Torres, A. (2007). Proceso de construcción social del espacio público Parque General Santander, ciudad de Florencia, Caquetá (Tesis de grado, 


\section{territarias 31} 204
Universidad Pedagógica Nacional, Bogotá, Colombia).

Parra, O., Ruiz, L., López, S., Torres, A., Gil, A., Arias, G. M.,... Idárraga, C. F. (2010). Espacio público y comercio en la calle. 'Ventas informales'. Experiencias Pereira-Colombia 2008-2010. Pereira: Universidad Católica Popular de Risaralda, Alcaldía de Pereira.

Pecha, P. (2006). Historia institucional del Instituto Distrital de Cultura y Turismo, 1978-2003. Bogotá: Alcaldía Mayor de Bogotá.

Pinto, N. (2010). Ciudadanía activa en la generación de nuevos espacios públicos en Bogotá (Tesis de grado, Universidad Piloto de Colombia, Bogotá, Colombia).

Plazas, J. (2006). Gestión social para el diseño de parques en el Distrito Capital (Tesis de grado, Universidad Piloto de Colombia, Bogotá, Colombia).

Plazas, M. (2006). Disposición y aceptación de una nueva cultura (tradición vs. implementación). Efectos de la intervención en el espacio público: 'el caso de la plaza de Lourdes y su cambio de identidad' (Tesis de grado, Universidad Piloto de Colombia, Bogotá, Colombia).

Rico, H. (2011). El Parque del Bicentenario (Tesis de grado, Universidad Pedagógica Nacional, Bogotá, Colombia).

Rincón, E. (2010). Experiencias urbanas en jóvenes: una posibilidad para el aprendizaje (Tesis de grado, Universidad Pedagógica Nacional, Bogotá, Colombia).

Rojas, I. (2010). Ocupación indebida de las áreas de antejardin: lineamientos hacia la garantía de aplicación de la función social y ecológica de la propiedad en el marco de la gestión urbana (Tesis de grado, Universidad Piloto de Colombia, Bogotá, Colombia).

Rosero, J. (2007). Evaluación de las cesiones de espacio público en los planes parciales adoptados para la ciudad de Bogotá (Tesis de grado, Universidad Piloto de Colombia, Bogotá, Colombia).

Salcedo, M. A. (2008a). El espacio público como objeto de estudio en las ciencias sociales y humanas. Revista Guillermo de Ockham, 6(1).

Salcedo, M. A. (2008b). Psicología, ciudad y espacio público. Revista Electrónica de Psicología Social, (15).

Salcedo, M. A. (2010a). Cali: educación, identidad y comportamiento ciudadano en el espacio público. Cali: Universidad de San Buenaventura.

Salcedo, M. A. (2010b). La cotidianidad dramática en el espacio público de dos cruces viales de la ciudad de Cali. Revista Ciencias Humanas, 7(1).

Saldarriaga, A., Rivadeneira, R., \& Jaramillo, S. (1998). Bogotá a través de las imágenes y las palabras. Bogotá: Tercer Mundo Editores y Observatorio de Cultura Urbana.

Secretaría de Cultura, Recreación y Deporte. (2010). Medicionesy sondeos. Recuperado de http://www.culturarecreacionydeporte.gov.co/observatorio/medAlparque.html

Secretaría de Cultura, Recreación y Deporte. (2011). Encuesta bienal de culturas. Resultados. Recuperado de http://www. culturarecreacionydeporte.gov.co/ 
observatorio/documentos/encuesta/ encuestal 1/d_resultados.html

Secretaría de Cultura, Recreación y Deporte.

(s. f.). ¿Qué es el Observatorio de Culturas? Recuperado de http://www.culturarecreacionydeporte.gov.co/observatorio/descargas/OBC.pdf

Secretaría de Cultura, Recreación y Deporte. (s. f.). Observatorio de Culturas. Recuperado de: http://www.culturarecreacionydeporte.gov.co/observatorio/ acercade.html

Sierra, S. P. (2011). Las zonas de transición de aprovechamientos autorizados como herramienta de gestión socioeconómica en el espacio público: análisis del programa 2008-2009 para el mejoramiento de sus procesos (Tesis de grado, Universidad Piloto de Colombia, Bogotá, Colombia).

Vidal, O. (2008). Impactos por el mal funcionamiento de los equipamientos y su entorno cuando se organizan eventos en dichas instalaciones (Tesis de grado,
Universidad Piloto de Colombia, Bogotá, Colombia).

Villamizar, A., \& Rondón, J. (2007). Caracterización del proceso de construcción social del espacio público. Plaza de mercado La Concordia de la ciudad de Florencia. Una mirada desde la pedagogía (Tesis de grado, Universidad Pedagógica Nacional, Bogotá, Colombia).

Yory, C. M. (2002). Del monumento a la ciudad. El fin de la idea de monumento en el nuevo orden espacio-temporal de la ciudad. Bogotá: Centro Editorial Javeriano (CEJA).

Yory, C. M. (Ed.). (2007). Espacio público y formación de ciudadanía. Bogotá: Editorial Pontificia Universidad Javeriana.

Zambrano, F., \& Museo de Desarrollo Urbano de Bogotá. (2001). Espacio público por la ciudad: exposición. Bogotá: Alcaldía Mayor de Bogotá, Defensoría del Espacio Público, Museo de Desarrollo Urbano. 
